\title{
Engineered 3D nanofibers by cold-plate electrospinning technique
}

\author{
Faheem A Sheikh* and Mushtaq Ahmad Beigh" \\ Department of Nano-Technology, University of Kashmir, Srinagar-190006, Jammu and Kashmir, \\ India \\ Email:* faheemnt@uok.edu.in, \# beighm@uok.edu.in
}

2D nanofibers produced using the traditional electrospinning technique results to have scaffolds with very small pores, thus limiting cellular infiltration and further limiting the tissue ingrowth in designed scaffolds. In this work, a facile strategy have successfully introduced that can produce 3D silk fibroin nanofibers (Figure 1), obviating an intrinsic limitation of traditional electrospinning, with high porosity and, most importantly, with controlled full-thickness using cold-plate electrospinning. Silk fibroin nanofibers fabricated by this technique presented a high porosity and a controlled full-thickness compared to the nanofibers prepared by traditional electrospinning and/or by saltleaching electrospinning process. The cell attachment and infiltration studies have been conducted to focus the rationale of using the $3 \mathrm{D}$ nanofibers by cold-plate electrospinning as a potential candidate to overcome drawbacks of electrospinning techniques. This technique can be directly applied to facilitate the fabrication of biomaterials with exact shapes and morphologies; in particular, such nanofibers are highly desirable for creating structures that resemble the dermis, nose, or ear or for repairing other facial defects.

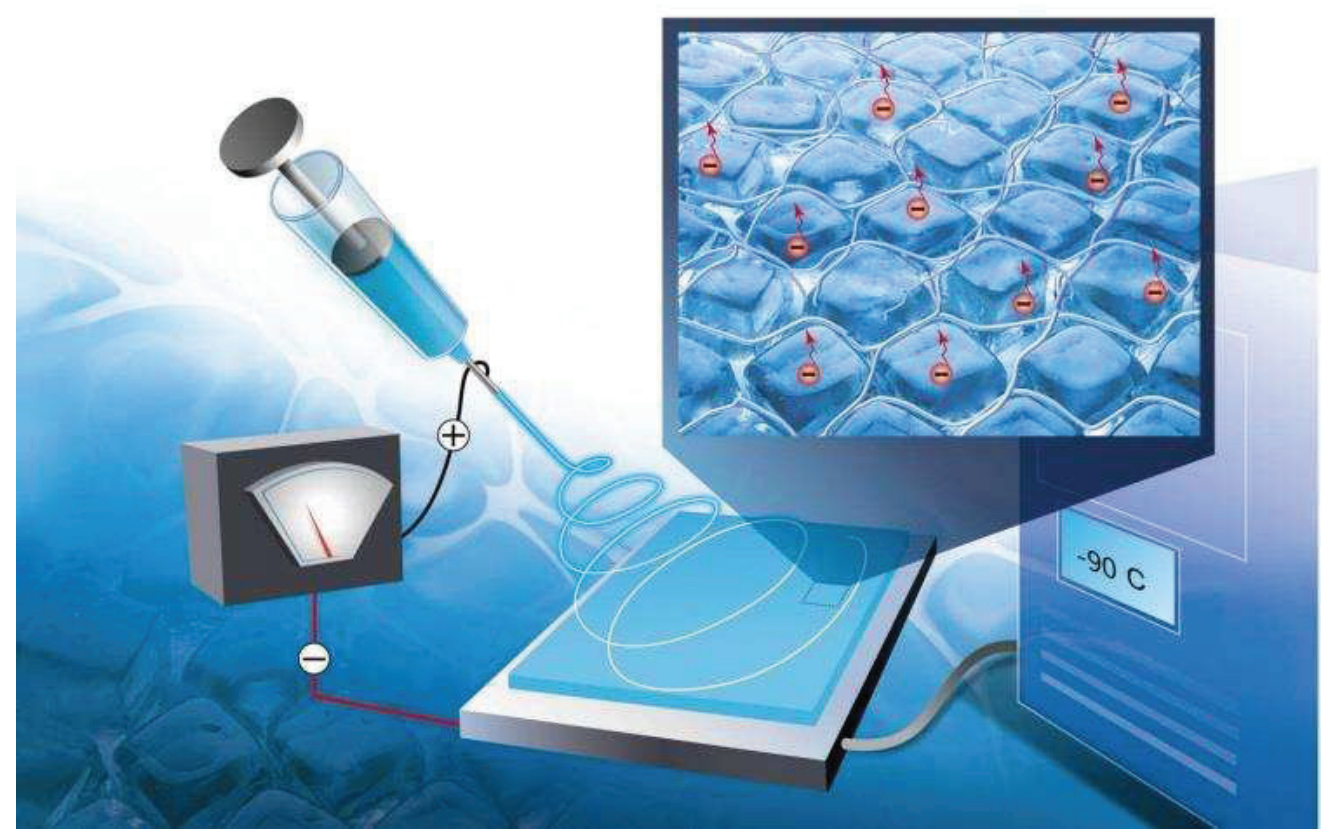

Figure 1: Schematic showing 3D electrospinning technique 